\title{
A new colour for optical chips
}

\author{
Microphotonic devices are becoming increasingly sophisticated. Nature Photonics spoke to \\ Michal Lipson from Cornell University about her team's recent success in adding a new type of \\ wavelength converter to the silicon-photonics toolbox.
}

What was the motivation for making these miniature wavelength converters? Wavelength conversion is used in large-scale optical networks to switch data between different channels and give the network increased flexibility and capacity. In our paper (page 293) we report that we have been able to do exactly the same thing but on the scale of a chip. In contrast to previous schemes, our approach has the potential to control the wavelength shift electrically, which is a big benefit for chip integration. In effect, we are taking the sophisticated features and the enormous bandwidth routinely used in the world of telecommunications and making it available for applications in optical interconnects. The hope is that this will potentially allow more advanced forms of data communication between silicon chips in the future.

How do your silicon-chip wavelength converters work?

We use silicon microring resonators that are just a few micrometres in diameter. The principle of operation is very similar to well-known resonance effects in the field of acoustics. If you think about a guitar, you can pluck one of the strings and it vibrates at a resonant frequency, which is defined by the length of the string, in other words the size of the cavity. Now, if while the string is vibrating and the sound is being generated you change the length of the cavity, by moving the position of your finger clamping the end of the string, you will change the frequency of the sound. We are doing the same thing but in the world of optics. We are letting light into a resonator (a silicon microring) and changing the effective length of the resonator while light is still inside. As a result, the colour or wavelength of the light readjusts itself to fit inside the resonator - in other words wavelength conversion. This change in the cavity's effective length is caused by injecting electrons and holes into the resonator, which increases the refractive index of the light path, equivalent to lengthening it. So far we have used an additional light beam to generate the electrons and the holes that are required,

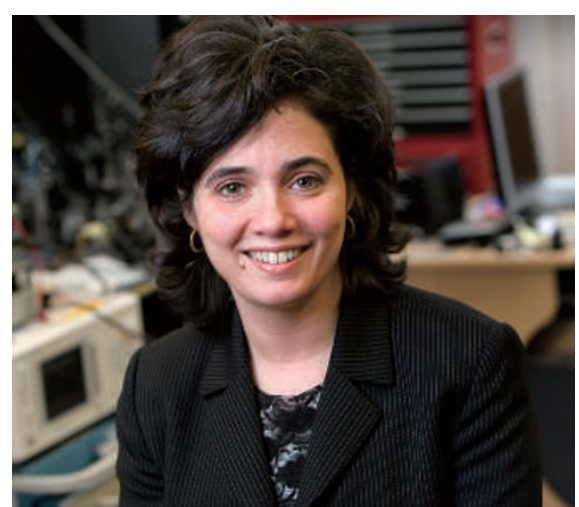

Michal Lipson in her silicon-photonics lab. Courtesy of Jason Koski, Cornell University Photography.

but a PIN-diode electrical structure could provide a direct means of doing this electrically at very high speeds.

So far you have reported wavelength shifts of 2-3 nm with a conversion efficiency of $30-35 \%$. Could this be improved? Absolutely, the numbers that we have reported are not fundamentally limited. We are working on optimizing the fabrication process and lowering the optical losses and designing structures that are more sensitive to ultrafast tuning mechanisms. One option is to cascade a sequence of these rings and connect them together. For example, in your first issue IBM showed that it is possible to cascade 50-100 rings, albeit for a different type of application.

What about the prospects for integrating these converters with other silicon photonic devices?

We are working very hard to create massively integrated systems, where we integrate many devices with different functionalities, such as wavelength conversion, buffers that hold or store light or ultrafast modulators, where you convert data from the electrical to the optical regime. We want to take systems and devices that are commonly found in optical networks and integrate them together on a single chip using silicon photonics.
How do you see the field of silicon microphotonics evolving over the next few years?

I think that this field is moving forward in two different directions - namely integration and performance. The first is concerned with improving the integration of devices on a common silicon platform and unifying the worlds of microelectronics and photonics. The second is dedicated to optimizing the performance of individual devices and designing systems that will exploit the enormous bandwidths they provide. There is also much work to be done in pursuing fundamental physics. Advanced silicon-fabrication techniques, such as high-resolution lithography and deep selective etching, allow us to make devices with extremely high precision, and now, for the first time, we can build structures that confine light very strongly in regions with dimensions as small as the wavelength of light or even smaller. This allows us to create devices that exhibit new physics - breaking the bandwidth time-delay limit for slow light, for example.

What challenges do you think will need to be overcome along the way? Massive integration on a chip is unbelievably challenging. Power consumption needs to be extremely small and devices, such as switches and modulators, need to operate at faster and faster data rates while maintaining their small size. At the same time we need to make sure that everything is compatible with current microelectronics and can be integrated together. You should not forget that silicon is not a natural optical material, so we are having to beat nature. The way that we are doing this is by making devices that strongly confine light and effectively enhance electro-optic and nonlinear effects.

Interview by Oliver Graydon.

Lipson and her co-workers have a letter on wavelength conversion in silicon resonators on p293 of this issue. 